\section{Câncer de mama: mortalidade crescente na Região Sul do Brasil entre 1980 e 2002}

\author{
Increase in breast cancer mortality in Southern \\ Brazil from 1980 to 2002
}

\footnotetext{
1 Faculdade de Medicina, Universidade Federal do Rio Grande do Sul, Porto Alegre, Brasil. 2 Instituto de Matemática, Universidade Federal do Rio Grande do Sul, Porto Alegre, Brasil.

3 Departamento de Educação Física e Saúde, Universidade de Santa Cruz do Sul, Santa Cruz do Sul, Brasil.

Correspondência

L. N. Nunes

Departamento de Estatística, Instituto de Matemática, Universidade Federal do Rio Grande do Sul. Av. Bento Gonçalves 9500 Porto Alegre, RS 91509-900, Brasil. lununes@mat.ufrgs.br
}

\begin{abstract}
Breast cancer is the most prevalent form of cancer in the world. Breast cancer mortality rates are high in Brazil and show striking variations between geographic regions. A time-trend ecological study was performed in Southern Brazil from 1980 to 2002. Data were collected from the Mortality Information System (Ministry of Health) to assess age-standardized mortality rates. Linear regression for mortality time-trend analysis and multiple regression for mortality differences among three States were calculated. The highest mean mortality rate (14.45) was observed in Rio Grande do Sul, significantly greater $(p<0.001)$ than in Santa Catarina (8.93) and Paraná (9.95). An annual increase of 0.47 in the mortality rate was observed in the three States of Southern Brazil. According to these results, the South of Brazil and especially the State of Rio Grande do Sul showed a significant upward trend in breast cancer mortality. Continued efforts are needed to help explain these numbers and reverse the present situation.
\end{abstract}

Breast Neoplasms; Mortality Rate; Women's Health

\author{
Andrea T. Cadaval Gonçalves 1 \\ Paulo Fernandes Costa Jobim 1 \\ Roberta Vanacor 1 \\ Luciana Neves Nunes 1,2 \\ Isabella Martins de Albuquerque 1,3 \\ Mary Clarisse Bozzetti 1
}

\section{Introdução}

Nas estimativas globais do ano de 2002 1, o câncer de mama é considerado o mais prevalente em todo o mundo, sendo também a principal causa de morte por câncer e o tipo mais freqüente entre as mulheres (23\% de todas as neoplasias). Estimam-se 1,15 milhão de novos casos no mundo, ocupando o segundo lugar geral quando ambos os sexos são considerados conjuntamente, e 411 mil mortes no mesmo período.

Observaram-se, nas últimas décadas, no Brasil e no mundo, importantes mudanças sóciodemográficas, com alteração da estrutura etária e das taxas de fecundação, com conseqüente envelhecimento populacional. Sabe-se que o risco de morte por câncer aumenta com a idade 2. Com isso, ocorreram modificações na distribuição dos óbitos segundo a causa básica de morte.

O Instituto Nacional de Câncer (INCA) estimou, em 49.470, o número de casos novos de câncer de mama no Brasil em 2005, com um risco estimado de 53 casos a cada 100 mil mulheres. $\mathrm{Na}$ Região Sudeste, o câncer de mama é o mais incidente entre as mulheres com um risco estimado de 73 casos novos por 100 mil. Sem considerar os tumores de pele não-melanoma, esse tipo de câncer também é o mais freqüente nas mulheres das regiões Sul (71/100 mil), Centro-Oeste (38/100 mil) e Nordeste $(27 / 100 \mathrm{mil}){ }^{3}$.

O prognóstico do câncer de mama é relativamente bom se diagnosticado nos estádios 
iniciais. Estima-se que a sobrevida média geral cumulativa, após cinco anos, seja de $65 \%$ nos países desenvolvidos e de 56\% nos países em desenvolvimento. Na população mundial, a sobrevida média, após cinco anos, é de $61 \%$. No Brasil, as taxas de mortalidade por câncer de mama continuam elevadas 2 .

Em relação ao diagnóstico precoce, ensaios clínicos randomizados têm demonstrado que a mamografia de rastreamento para mulheres de 40 a 79 anos pode reduzir a mortalidade, sendo o maior impacto encontrado na faixa etária de 50 a 69 anos, com redução da mortalidade em torno de $25 \%$ nesse grupo 4 . Embora esses achados tenham sido contestados em algumas metanálises recentemente publicadas 5,6.

Além da idade, história prévia ou familiar de câncer de mama ou ovário, biópsias de mama e da presença de mutação nos genes BRCA 1 e 2 , vários outros fatores de risco têm sido levantados 6,8. Entretanto, não existem medidas práticas específicas de prevenção primária do câncer de mama, aplicáveis à população. Estudos observacionais têm sugerido que a prevenção do tabagismo, do alcoolismo, da obesidade e do sedentarismo e o incentivo ao aleitamento materno reduzem o risco de câncer de mama 2 . No entanto, os avanços tecnológicos mais importantes têm sido direcionados para o diagnóstico precoce e para o tratamento 2,5,8.

O objetivo deste trabalho é avaliar o comportamento do coeficiente de mortalidade por câncer de mama em mulheres nos três Estados da Região Sul do Brasil, padronizado por idade, utilizando um modelo de série temporal, entre os anos de 1980 a 2002.

\section{Métodos}

Foi realizado um estudo ecológico de série temporal, sendo coletados dados anuais do Departamento de Informação e Informática do SUS (DATASUS, http://www.datasus.gov.br), para o período entre 1980 a 2002, da Região Sul, por Unidade da Federação dos Estados do Rio Grande do Sul, Santa Catarina e Paraná. Foram calculadas as taxas de mortalidade por 100 mil habitantes do sexo feminino por ano e para cada Estado. Essas taxas foram padronizadas por faixas etárias, pelo método direto 9, utilizando-se, como referência, os dados de população residente em cada Estado, sendo esses obtidos por meio de pesquisa do DATASUS, referentes ao Censo Demográfico 2000. Foram utilizadas cinco faixas etárias, 20-29 anos, 30-39 anos, 40-49 anos, 50-59 e 60-69 anos. Para a análise estatística, utilizou-se modelo de regressão linear simples, a fim de estimar os coeficien- tes das taxas de mortalidade padronizadas em cada um dos Estados. Com o intuito de comparar as diferenças entre os três Estados, utilizou-se um modelo de regressão linear múltipla, em que se considerou, como variável dependente, a taxa de mortalidade padronizada e, como variáveis independentes, o tempo e os Estados.

Para todos os modelos, foram feitos diagnósticos pela análise de resíduos. Os dados obtidos foram tratados nos programas Microsoft Excel 2000 (Microsoft Corp., Estados Unidos) e SPSS 12.0 (SPSS Inc., Chicago, Estados Unidos). Para as análises estatísticas, o nível de significância utilizado foi de $5 \%$.

\section{Resultados}

A Região Sul do Brasil, formada pelos Estados do Rio Grande do Sul, Paraná e Santa Catarina, tem os dados de áreas geográficas, populações e taxas médias de mortalidade por câncer de mama, padronizadas para idade, apresentados conforme Tabela 1. Pela Figura 1, pode-se perceber que o Rio Grande do Sul apresenta maior taxa de mortalidade padronizada em todos os anos investigados, quando comparado com os Estados de Santa Catarina e Paraná, sendo que o Rio Grande do Sul já parte de um patamar mais elevado que os demais. Já os Estados de Santa Catarina e Paraná têm taxas de mortalidade bastante semelhantes.

Para todos os Estados, foram ajustadas retas de regressão linear simples, e a tendência é de aumento da taxa de mortalidade nos três locais, pois os coeficientes de inclinação, nas três retas, foram positivos e significativos (Figura 1). Ou seja, espera-se um aumento de 0,42, 0,41 e 0,47 óbito a cada ano na taxa de mortalidade por câncer de mama, respectivamente, nos Estados do Rio Grande do Sul, Santa Catarina e Paraná. Os ajustes dos modelos foram consistentes quando se verificaram os coeficientes de determinação $\left(R^{2}\right)$, sendo esses $0,96,0,95$ e 0,98 , respectivamente, para Rio Grande do Sul, Santa Catarina e Paraná.

O modelo $\left(\right.$ taxa $=3,95+0,47^{*}$ tempo + 5,42Rio Grande do Sul + 0,36*Santa Catarina 0,05*tempo*Rio Grande do Sul - 0,05tempo*Santa Catarina) foi obtido por meio da regressão linear múltipla, e somente a constante e os coeficientes de tempo e Rio Grande do Sul foram significativos. Portanto, é possível dizer que o Rio Grande do Sul é significativamente diferente de Santa Catarina e Paraná ( $\mathrm{p}<0,001)$, o que é equivalente a dizer que a taxa média de mortalidade do Rio Grande do Sul (14,45; Tabela 1) é diferente das taxas médias de Santa Catarina e Paraná (8,93 e 
Superfície territorial, população e população feminina de 20-69 anos e taxa média de mortalidade por Estado e Região Sul, Brasil, 2000.

\begin{tabular}{|c|c|c|c|c|}
\hline Estados & $\begin{array}{l}\text { Superfície territorial } \\
\qquad\left(\mathrm{km}^{2}\right)^{*}\end{array}$ & População ** & $\begin{array}{l}\text { População feminina } \\
\text { (20-69 anos) ** }\end{array}$ & $\begin{array}{c}\text { Taxa média } \\
\text { de mortalidade }\end{array}$ \\
\hline Rio Grande do Sul & 281.748 & 10.187 .798 & 3.131 .587 & 14,45 \\
\hline Santa Catarina & 95.346 & 5.356 .360 & 1.579 .236 & 8,93 \\
\hline Paraná & 199.314 & 9.563 .458 & 2.828 .121 & 9,95 \\
\hline Total & & 25.107 .616 & & 11,11 \\
\hline
\end{tabular}

* Instituto Brasileiro de Geografia e Estatística 12;

** Departamento de Informática do SUS (DATASUS; http://www.datasus.gov.br).

Figura 1

Tendência da taxa de mortalidade, ajustada para tempo, para câncer de mama, nos Estados do Rio Grande do Sul, Santa Catarina e Paraná, Brasil, no período de 1980 a 2002

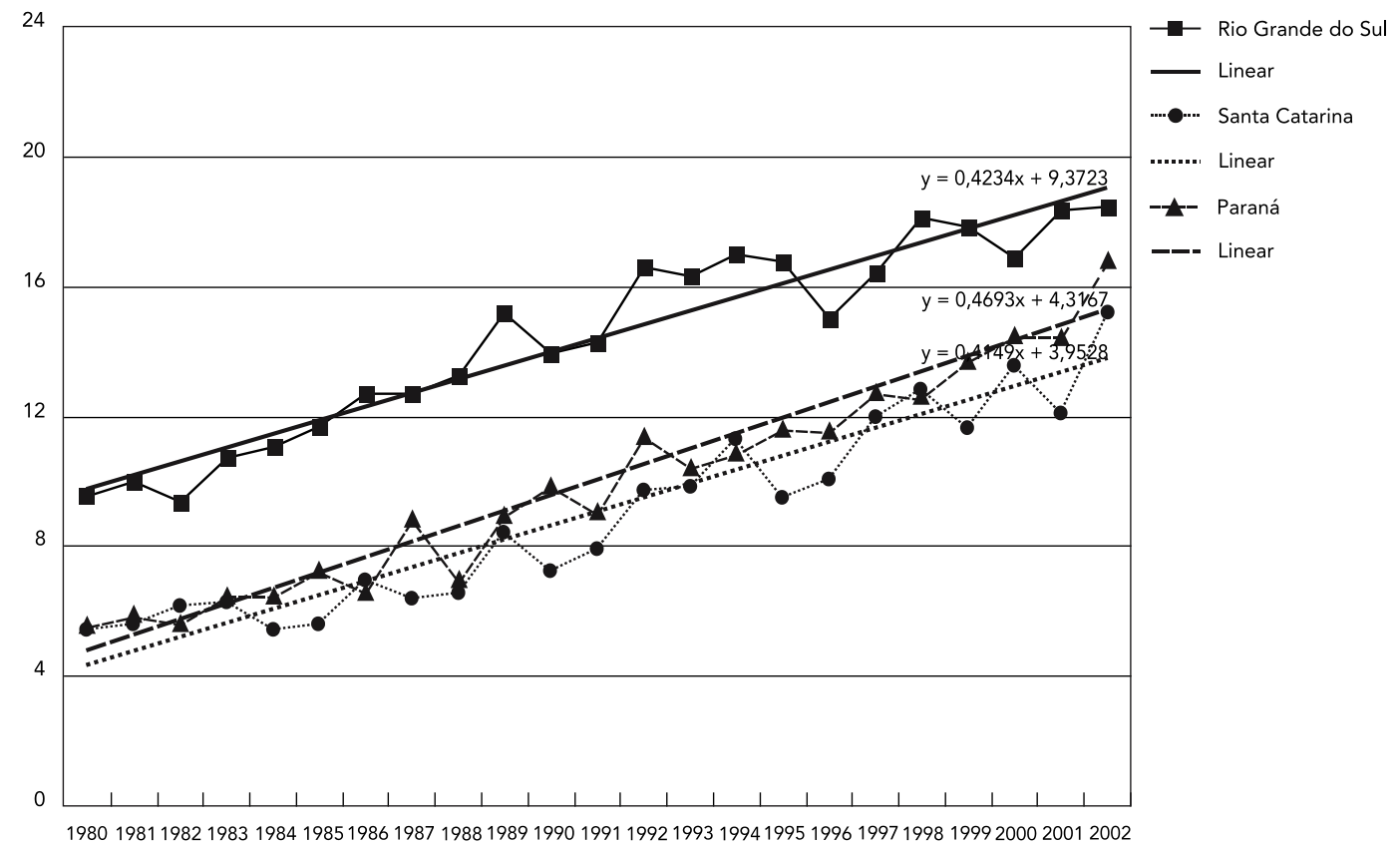

9,95; respectivamente). Quando ajustado para os Estados, o coeficiente do tempo foi significativo ( $p<0,001)$, portanto estima-se que, a cada ano, haja um aumento de 0,47 óbito na taxa de mortalidade por câncer de mama, independente do Estado. Não houve interação entre tempo e Estados, observando-se que as retas de regressão são paralelas (Figura 1), ou seja, a inclinação é aproximadamente a mesma para todos os Estados. O coeficiente de determinação do modelo múltiplo foi 0,98 , indicando um ótimo ajuste do modelo.

\section{Discussão}

Os resultados indicam que há tendência de aumento da mortalidade por câncer de mama nos três Estados da Região Sul do Brasil, com taxa de 
0,47 de aumento de óbitos por ano, independente do Estado. É importante ressaltar que o Rio Grande do Sul parte de um patamar mais elevado, apresentando uma taxa média $(14,45)$ de mortalidade significativamente maior que os outros dois Estados $(\mathrm{p}<0,001)$. Esses dados podem ser conseqüentes à tendência crescente da incidência dessa doença associada à deficiência no diagnóstico precoce, terapêutica e seguimento inadequados 10. Entretanto, os potenciais fatores determinantes dessas diferenças encontradas entre os três Estados avaliados neste estudo são de difícil identificação na literatura 3 .

Observa-se, no Brasil e no mundo, aumento da incidência do câncer de mama. De acordo com as estimativas do ano de 2005, apresentadas pelo INCA, a taxa bruta por 100 mil habitantes no Rio Grande do Sul $(91,43)$ representa quase o dobro que Santa Catarina $(54,88)$ e Paraná $(57,72)^{3}$. De acordo com Parkin et al. 1, as estimativas globais exibem que a incidência dessa doença, na maioria dos países, supera algumas previsões, com um aumento anual de cerca de $0,5 \%$ desde 1990. Entretanto, existe uma variabilidade entre as taxas de mortalidade por câncer de mama no mundo, observando-se redução da mortalidade nos países desenvolvidos e aumento nos países em desenvolvimento, como é o caso do Brasil 1. Sabe-se que, diferente de alguns países desenvolvidos, o Brasil não dispõe de um programa organizado de rastreamento do câncer de mama.

A Região Sul compreende 7\% do espaço territorial total do Brasil, exibe altos níveis de industrialização, e sua população revela os melhores indicadores de qualidade de vida do país. O Rio Grande do Sul é o Estado brasileiro com maior expectativa de vida $(73,4$ anos), superior à média brasileira de 69,0 anos. Os idosos ( $\geq 60$ anos) representam aproximadamente $10 \%$ da população 11. Entre 1980 e 1995, a mortalidade feminina por câncer de mama aumentou em todas as regiões, mas o risco de morte no Sul e no Sudeste é pelo menos duas vezes maior do que nas outras regiões ${ }^{12}$. Esses dados levam a crer que a carência de um programa de rastreamento numa população, na qual se observa envelhecimento e melhor qualidade de vida, pode ser uma das hipóteses para explicar os achados do presente estudo.

Parece haver semelhança nas taxas de incidência de câncer de mama na Região Sul do Brasil com grande parte dos países da Europa 13. Existem alguns possíveis fatores de risco em comum, observados nessas regiões, como o predomínio da raça branca, menor número médio de filhos, gestações iniciadas em idades mais avançadas, melhor nível socioeconômico e maior uso de terapia de reposição hormonal 12,14. Contudo, as taxas de incidências de câncer de mama são mais elevadas no Rio Grande do Sul do que nos outros dois Estados do Sul do Brasil 3, e essas diferenças podem refletir heterogeneidade de perfis de exposição a fatores de risco e de modos de vida e fatores genéticos ainda não totalmente identificados. Entretanto, até o momento, não existem dados consistentes na literatura para esclarecer tais suposições.

Porto Alegre, capital do Rio Grande do Sul, registra a mais alta incidência de câncer de mama quando comparada às demais capitais da Região Sul e do país. De acordo com dados do INCA 3, em Porto Alegre, a taxa bruta de incidência estimada para 2005 é de 151,66 em 100 mil mulheres, já para Florianópolis (Santa Catarina), 87,81 e Curitiba (Paraná), 85,75. Observaram-se também dados de óbito por ocorrência mais elevados nas capitais da Região Sul do que óbitos por residência. Isso revela, muito provavelmente, a significativa tendência à transferência de pacientes mais graves ou mesmo em fases terminais para o atendimento médico nas capitais.

O prognóstico do câncer de mama é bom especialmente se diagnosticado em fases precoces (estágios I e II) e tratado de acordo com os protocolos atuais. Porém, no Brasil, cerca de metade dos tumores de mama são diagnosticados nos estágios III e IV 7, o que pode estar diretamente relacionado à falta de um programa de rastreamento que inclua a realização de exame clínico das mamas, mamografia anual e a identificação de grupos populacionais com risco elevado para o desenvolvimento do câncer de mama. A mamografia, como método para rastreamento, é uma medida de impacto na redução da mortalidade, especialmente em mulheres de 50 a 69 anos 15. Sabe-se ainda que, além da necessidade de médico radiologista habilitado para a interpretação de laudos mamográficos, a qualidade técnica desse exame é fundamental para a redução dos casos de falsos negativos 16 .

O Inquérito Domiciliar sobre Comportamentos de Risco e Morbidade Referida de Doenças e Agravos não Transmissíveis, do Ministério da Saúde, desenvolvido pelo INCA em parceria com a Secretaria de Vigilância em Saúde, mostrou que, para as 16 localidades brasileiras analisadas (15 capitais e o Distrito Federal), a cobertura estimada de realização de mamografia variou entre $37 \mathrm{e}$ $76 \%$. Entretanto, o percentual de realização desse exame pelo SUS variou entre 17 e $54 \%$ do total 3 . A cobertura insuficiente e a dificuldade no controle e na avaliação dos serviços de mamografia disponíveis pelo SUS retardam o diagnóstico do câncer de mama.

Em relação ao tratamento do câncer de mama, a maioria das pacientes pode ser submetida a cirurgias conservadoras, radioterapia local, te- 
rapias hormonais e quimioterapia, resultando em redução de mortalidade em torno de 25 a $50 \% 17$. Observa-se que, cada vez mais, surgem novas opções, como, por exemplo: o uso de tamoxifeno profilático para pacientes de alto risco, novos agentes citotóxicos e progressos no entendimento da biologia tumoral que permitem a identificação de tumores mais agressivos ou resistentes à quimioterapia 16. É evidente que esses avanços não estão disponíveis a toda a população. De acordo com estatísticas norteamericanas, a mortalidade em afro-americanas é maior que nas brancas. No entanto, quando o tratamento recebido é semelhante, essa disparidade desaparece 18. A diferença encontrada nas taxas de mortalidade entre os estados e a tendência ao aumento na mortalidade nos três estados referidos no presente estudo também podem estar relacionadas a variações dos tratamentos oferecidos a essas pacientes. Estudos avaliando a efetividade da terapêutica do câncer

\section{Resumo}

O câncer de mama é apontado como o tipo de câncer mais prevalente no mundo. No Brasil, as taxas de mortalidade por câncer de mama continuam elevadas, observando-se diferenças inter-regionais. Foi realizado um estudo ecológico de série temporal (1980-2002) na Região Sul, com dados anuais do Departamento de Informação e Informática do SUS (DATASUS), para avaliar o comportamento do coeficiente de mortalidade por câncer de mama, padronizado por idade. Utilizou-se regressão linear simples e múltipla para estimar as taxas de mortalidade e as diferenças entre os três Estados. O Rio Grande do Sul parte de um patamar mais elevado e apresenta maior taxa média de mortalidade $(14,45)$, sendo significativamente diferente ( $p<0,001)$ quando comparado com Santa Catarina $(8,93)$ e Paraná $(9,95)$. Observou-se um aumento anual de 0,47 óbito na taxa de mortalidade por câncer de mama, independente do Estado. Conclui-se que há uma tendência similar de aumento da mortalidade por câncer de mama nos três Estados da Região Sul, com índices significativamente maiores no Rio Grande do Sul, enfatizando-se a importância da identificação dos fatores relacionados a esse quadro alarmante e o estabelecimento de medidas efetivas a fim de reverter esses números.

Neoplasias Mamárias; Coeficiente de Mortalidade; Saúde da Mulher de mama utilizada na Região Sul bem como do seguimento dessas pacientes após o tratamento adjuvante são fundamentais.

A Região Sul, em especial o Rio Grande do Sul, exibe estatísticas alarmantes e com tendência a piora na mortalidade do câncer de mama. Ao contrário de alguns países desenvolvidos, percebemos que o Estado que apresenta a maior incidência de câncer de mama, o Rio Grande do Sul, é justamente aquele com maior taxa média de mortalidade por essa doença. Esses dados apontam para a necessidade urgente de novas pesquisas que elucidem os fatores determinantes da alta incidência e da elevada mortalidade nessas regiões.

Além disso, a demonstração de incidência e mortalidade crescentes, em diferentes proporções nos Estados de uma mesma região, é de grande importância para o direcionamento de estratégias e políticas de assistência à saúde para o controle do câncer de mama no Brasil.

\section{Colaboradores}

A. T. C. Gonçalves participou da concepção do estudo, da discussão dos resultados, da redação e da revisão do manuscrito. P. F. C. Jobim contribuiu na concepção do estudo, coleta dos dados, redação e revisão do manuscrito. L. N. Nunes colaborou na concepção do estudo, coleta de dados, análise dos dados, redação dos resultados e revisão do manuscrito. R. Vanacor e I. M. Albuquerque participaram da concepção do estudo, da redação e revisão do manuscrito. M. C. Bozzetti contribuiu na concepção do estudo e revisão do manuscrito. 


\section{Referências}

1. Parkin MD, Bray F, Ferlay J, Pisani P. Global cancer statistics, 2002. CA Cancer J Clin 2005; 55:74-108.

2. Curi Hallal AL, Gotlieb SLD, Latorre MRDO. Evolução da mortalidade por neoplasias malignas no Rio Grande do Sul, 1979-1995. Rev Bras Epidemiol 2001; 4:169-77.

3. Instituto Nacional de Câncer. Estimativa 2005: incidência de câncer no Brasil. http://www.inca. gov.br/estimativa/2005/versaofinal.pdf (acessado em 06/Set/2005).

4. Nyström L, Andersson I, Bjurstam N, Frisell J, Nordenskjold B, Rutqvist LE. Longterm effects of mammography screening: updated overview of the Swedish randomized trials. Lancet 2002; 359:90919.

5. Olsen O, Gøtzsche PC. Cochrane review on screening for breast cancer with mammography. Lancet 2001; 358:1340-2.

6. Fletcher SW, Elmore JG. Clinical practice: mammographic screening for breast cancer. N Engl J Med. 2003; 348:1672-80.

7. Struewing JP, Hartge P, Wacholder S, Baker SM, Berlin M, McAdams M, et al. The risk of cancer associated with specific mutations of BRCAl and BRCA2 among Ashkenazi Jews. N Engl J Med 1997; 336:1401-8.

8. Schwartsmann G. Breast cancer in South America: challenges to improve early detection and medical management of a public health problem. J Clin Oncol 2001; 19(18 Suppl):118S-24S

9. Schoenbach JV. Understanding the fundamentals of epidemiology: an evolving text. http://www. epidemiolog.net/evolving/ (acessado em 19/Set/ 2005).
10. Moraes AB, Zanini RR, Turchiello MS, Riboldi J, Medeiros LR. Estudo da sobrevida de pacientes com câncer de mama atendidas no hospital da Universidade Federal de Santa Maria, Rio Grande do Sul, Brasil. Cad Saúde Pública 2006; 22: 221928.

11. Fundação de Economia e Estatística. Expectativa de vida, por sexo, no Brasil e no Rio Grande do Sul - 1992 e 2003. http://www.fee.tche.br/sitefee/ $\mathrm{pt} /$ content/resumo/pg_estado_tabela.php?id=10 (acessado em 17/Set/2005).

12. Instituto Brasileiro de Geografia e Estatística. Área territorial oficial: consulta por Unidade da Federação. http://www.ibge.gov.br/home/geociencias/ areaterritorial/principal.shtm (acessado em 17/ Set/2005).

13. Ward E, Jemal A, Cokkinides V, Singh GK, Cardinez C, Ghafoor A, et al. Cancer disparities by race/ethnicity and socioeconomic status. CA Cancer J Clin 2004; 54:78-93.

14. Wünsch Filho V, Moncau JE. Mortalidade por câncer no Brasil 1980-1995: padrões regionais e tendências temporais. Rev Assoc Med Bras 2002; 48:250-7.

15. Elmore JG, Armstrong K, Lehman CD, Fletcher SW. Screening for breast cancer. JAMA 2005; 293:124556.

16. Taplin SH, Rutter CM, Finder C, Mandelson MT, Houn F. White E. Screening mammography: clinical image quality and the risk of interval breast cancer. AJR Am J Roentgenol 2002; 178:797-803.

17. Hortobagyi GN. Treatment of breast cancer. N Engl J Med 1998; 339:974-84.

18. Wojcik BE, Spinks MK, Optenberg SA. Breast carcinoma survival analysis for African American and white women in an equal-access health care system. Cancer 1998; 82:1310-8.

Recebido em 25/Nov/2005

Versão final reapresentada em 24/Jan/2007 Aprovado em 07/Fev/2007 